hep-th/0509132

PUPT-2174

ITEP-TH-56/05

\title{
Perturbative Gauge Theory and Closed String Tachyons
}

\author{
A. Dymarsky ${ }^{1}$, I.R. Klebanov ${ }^{1}$ and R. Roiban ${ }^{1,2}$ \\ 1 Joseph Henry Laboratories, Princeton University, Princeton, NJ 08544, USA \\ ${ }^{2}$ Physics Department, Pennsylvania State University, University Park, PA 16802, USA
}

\begin{abstract}
We find an interesting connection between perturbative large $N$ gauge theory and closed superstrings. The gauge theory in question is found on $N$ D3-branes placed at the tip of the cone $\mathbb{R}^{6} / \Gamma$. In our previous work we showed that, when the orbifold group $\Gamma$ breaks all supersymmetry, then typically the gauge theory is not conformal because of doubletrace couplings whose one-loop beta functions do not possess real zeros. In this paper we observe a precise correspondence between the instabilities caused by the flow of these doubletrace couplings and the presence of tachyons in the twisted sectors of type IIB theory on orbifolds $\mathbb{R}^{3,1} \times \mathbb{R}^{6} / \Gamma$. For each twisted sectors that does not contain tachyons, we show that the corresponding double-trace coupling flows to a fixed point and does not cause an instability. However, whenever a twisted sector is tachyonic, we find that the corresponding one-loop beta function does not have a real zero, hence an instability is likely to exist in the gauge theory. We demonstrate explicitly the one-to-one correspondence between the regions of stability/instability in the space of charges under $\Gamma$ that arise in the perturbative gauge theory and in the free string theory. Possible implications of this remarkably simple gauge/string correspondence are discussed.
\end{abstract}




\section{Introduction}

A class of generalizations of the maximally supersymmetric $\mathrm{AdS}_{5} / \mathrm{CFT}_{4}$ duality [1, 2, 3] (see [4, 5. for reviews) is obtained through modding out both sides by a discrete subgroup $\Gamma$ of the $S U(4)$ R-symmetry [6, 7]. In this fashion one obtains quiver gauge theories with $\mathcal{N}=2$ supersymmetry for $\Gamma \subset S U(2)$, with $\mathcal{N}=1$ supersymmetry for $\Gamma \subset S U(3)$, and with no supersymmetry for all other $\Gamma$. In the large $N$ limit, correlation functions of the single-trace operators uncharged under the orbifold group are "inherited" from the parent $\mathcal{N}=4 \mathrm{SYM}$ theory: they are the same up to a rescaling of the " $t$ Hooft coupling $\lambda=g_{Y M}^{2} N$ [ 8 , 9 . In particular, the beta functions for marginal single-trace operators vanish in the large $N$ limit. However, there is no such simple "inheritance" for the twisted operators that are charged under the orbifold group $\Gamma$.

The operators of particular interest to us are double-trace operators $O^{g} O^{g^{\dagger}}$, where $O^{g}$ is a twisted single-trace operator corresponding to the element $g$ of the orbifold group $\Gamma$. Even though such operators have no net charge, they are not protected by the "inheritance principle" [8, 9]. Indeed, beta functions for marginal operators of this type, made of scalar fields, are induced at one-loop order and are found to be proportional to $\lambda^{2}$ [10, 11, 12]. However, as shown in our previous work [13], this does not necessarily imply that the large $N$ conformal invariance is lost: an induced double-trace coupling may flow to a fixed point.

A systematic procedure for studying the issue of conformal invariance, that was used in [13, is to add each induced marginal double-trace operator to the action,

$$
\delta S=-\int d^{4} x f O \bar{O} .
$$

The complete one-loop beta function in such a theory has the general structure

$$
M \frac{\partial f}{\partial M}=\beta_{f}=v_{O} f^{2}+2 \gamma_{O} \lambda f+a_{O} \lambda^{2} .
$$

It is crucial that the right hand side is not suppressed by powers of $N$. On the other hand, the beta function for $\lambda$ has no such leading large $N$ contribution, due to the theorem of 8. 9. ${ }^{1}$ Therefore, in the large $N$ limit, $\lambda$ may be dialed as we wish. In particular, it can be made very small so that the one-loop approximation in (2) is justified. Then the equation $\beta_{f}=0$ has two solutions, $f=a_{ \pm} \lambda$, where

$$
a_{ \pm}=\frac{1}{v_{O}}\left(-\gamma_{O} \pm \sqrt{D}\right), \quad D=\gamma_{O}^{2}-a_{O} v_{O}
$$

If the discriminant $D$ is non-negative, then these solutions are real, so that $f$ may flow to an IR stable fixed point. If $D$ is negative, then there is no real fixed point for $f$, and this

\footnotetext{
${ }^{1}$ Counting the powers of $N$, and using charge conservation, one can show that the double-trace operators made of twisted single-trace ones cannot induce any planar beta functions for untwisted single-trace couplings.
} 
coupling is not stabilized at least in the one-loop approximation. In the orbifold examples considered in [13], some double-trace operators were found to have $D \geq 0$, and hence were stabilized. Others were found to have a negative $D$, and hence caused an instability of the weakly coupled gauge theory. No non-supersymmetric orbifolds were found in [13] such that all double-trace couplings were stabilized, raising a question whether any such gauge theories flow to a fixed line passing through the origin. It is natural to conjecture that the closed string dual of this instability is associated with tachyonic modes from the twisted sector of the orbifold $A d S_{5} \times S^{5} / \Gamma\left[12\right.$, 13]. For a $\Gamma$ that does not act freely on the $S^{5}$, the tachyons are present for all radii. Recalling that $R_{A d S}^{4} \sim \alpha^{2} \lambda$, we then expect the instability in the dual field theory to be present for all $\lambda$. More interesting examples are provided by the freely acting orbifolds where the twisted sector closed strings are highly stretched at large $\lambda$, so that the tachyons are lifted [12]. In [13] it was suggested that, for $\lambda$ smaller than some critical value, some closed string modes become tachyonic, causing an instability that is manifested in the gauge theory by the negative discriminant $D$ of the beta function.

Making such a correspondence between perturbative gauge theory and closed string tachyons precise appears to be a formidable task. The usual AdS/CFT correspondence seems to require calculations in highly curved space with RR background fields, a problem that has not yet been solved. In this paper we carry out a much simpler calculation on the closed string side, computing the spectrum of twisted closed strings on $\mathbb{R}^{3,1} \times \mathbb{R}^{6} / \Gamma$, i.e. in the limit where the backreaction of the D3-branes is ignored. Remarkably, we find that, whenever a given twisted sector contains a tachyon, the discriminant $D$ of the beta function of the corresponding double-trace coupling in the gauge theory is negative; conversely, when the zero-point energy in a given twisted sector vanishes, then $D$ is non-negative. ${ }^{2}$ We demonstrate, for general orbifold groups $\Gamma$, the one-to-one correspondence between the regions of stability/instability in the space of charges under $\Gamma$ that arise in the perturbative gauge theory and in the free string theory. This constitutes a remarkably simple correspondence between perturbative gauge theory and free closed strings, which holds in the absence of supersymmetry. We speculate on reasons for why this duality is at work in the Discussion section. Using the results on the region of instability for double-trace couplings, we prove that there are no non-supersymmetric orbifold gauge theories possessing a fixed line passing through the origin of the coupling constant space. This answers a question posed in our previous paper [13]. ${ }^{3}$

On the way to deriving these main results, we also tie some loose ends left over from our previous work [13. We show that removing the $U(1)$ factors present in the $U(N)^{k} / U(1)$

\footnotetext{
${ }^{2} \mathrm{~A}$ similar connection between closed string tachyons and instabilities of non-commutative gauge theories on D3-brances at orbifold singularities was made in [14.

${ }^{3}$ An investigation of other candidate non-supersymmetric large $N$ theories, such as those suggested in [15], will be reported on in a separate publication.
} 
quiver theories does not change the leading large $N$ beta function calculations carried out there using the orbifold projection methods. The only modification is caused by the appearance of double-trace terms in the tree-level action when the $U(1)$ 's are removed. In the case of the supersymmetry preserving orbifolds, we find that for the operators induced at one-loop level the discriminant vanishes and the double-trace beta functions have the form $(f+a \lambda)^{2}$, i.e. the coupling $f$ is stabilized at $-a \lambda$. This coefficient of the double-trace term in the action is in precise agreement with the value found through removing the $U(1)$ 's at tree level. This confirms the superconformal nature of the supersymmetric $S U(N)^{k}$ orbifold gauge theories.

\section{Double-trace corrections for general orbifolds}

In this section we review the results of [13] on the marginal double-trace operators in the 1-loop effective action of orbifold gauge theories. We will denote by $\Gamma \subset S U(4)$ the orbifold group and by $g$ the representation of the elements of $\Gamma$ in $S U(|\Gamma| N)$ where it acts by conjugation. The representation of $\Gamma$ in the spinor and vector representation of $S O(6) \sim S U(4)$ will be denoted by $r_{g}$ and $R_{g}$, respectively. Since the vector representation of $S O(6)$ is the 2-index antisymmetric tensor representation of $S U(4)$, we have $R_{g}=r_{g} \otimes r_{g}$. The scalar fields invariant under the orbifold group satisfy

$$
\phi^{I}=R_{g}^{I J} g \phi^{J} g^{\dagger}
$$

- The contribution of the fermion loop to the double-trace part of the effective action is:

$$
\begin{aligned}
& \delta S_{\text {Fermi }}^{1 \text { loop } \mid 2 \text { tr }}= \\
& =\lambda^{2} \frac{\operatorname{Div}}{|\Gamma|} \sum_{g \in \Gamma} \operatorname{Tr}\left[\gamma^{I} \gamma^{J} \gamma^{K} \gamma^{L} r_{g}\right]\left[2 \operatorname{Tr}\left(\phi^{J} \phi^{I} g^{\dagger}\right) \operatorname{Tr}\left(\phi^{K} \phi^{L} g\right)+\operatorname{Tr}\left(\phi^{K} \phi^{I} g^{\dagger}\right) \operatorname{Tr}\left(\phi^{J} \phi^{L} g\right)\right]
\end{aligned}
$$

where $\gamma^{I}$ denote the chiral (i.e. $4 \times 4$ ) 6-dimensional Dirac matrices. Our notation for the divergent part of a generic 1-loop scalar amplitude is

$$
\operatorname{Div}=\int \frac{d^{4} k}{(2 \pi)^{4}} \frac{1}{k^{4}}=\frac{1}{16 \pi^{2}} \ln \frac{\Lambda^{2}}{M^{2}}
$$

where $\Lambda$ is the UV cutoff and $M$ is the renormalization scale.

- The contribution of the vectors, scalars and ghost loops to the double-trace part of the effective action is:

$$
\delta S_{\text {Bose, ghost }}^{1 \text { loop } 2 \text { tr }}=-\lambda^{2} \frac{\operatorname{Div}}{2|\Gamma|} \sum_{g \in \Gamma}\left\{\left(\operatorname{Tr}\left[R_{g}\right]+2\right)\left(\operatorname{Tr}\left(\phi^{2} g^{\dagger}\right) \operatorname{Tr}\left(\phi^{2} g\right)+2 \operatorname{Tr}\left(\phi^{I} \phi^{J} g^{\dagger}\right) \operatorname{Tr}\left(\phi^{J} \phi^{I} g\right)\right)\right.
$$




$$
\begin{aligned}
& +2\left(R_{g}^{K Q}+\left(R_{g}^{-1}\right)^{K Q}\right) \operatorname{Tr}\left(\left[\phi^{I}, \phi^{Q}\right] g^{\dagger}\right) \operatorname{Tr}\left(\left[\phi^{I}, \phi^{K}\right] \gamma\right) \\
& \left.-2\left(\delta^{K I}\left(R_{g}^{-1}\right)^{P Q}+\delta^{P Q} R_{g}^{K I}-2 \delta^{P Q} \delta^{K I}\right) \operatorname{Tr}\left(\phi^{P} \phi^{Q} g^{\dagger}\right) \operatorname{Tr}\left(\phi^{K} \phi^{I} g\right)\right\} .
\end{aligned}
$$

- We see that double-trace operators made out of twisted single-trace operators are generated at 1-loop. Perturbative renormalizability requires then that they be added to the tree level action. Such a tree-level deformation

$$
\delta_{2 \text { trace }} S=\frac{1}{2} \sum_{g \in \Gamma} f_{g} O_{g}^{I J} O_{g^{\dagger}}^{J I} \quad \text { with } \quad O_{g}^{I J}=\operatorname{Tr}\left(g \phi^{I} \phi^{J}\right) .
$$

brings the following additional contributions to the effective action:

$$
\begin{gathered}
\delta S_{2 \text { trace }}^{1 \text { loop } \mid 2 \text { tr }}=-\frac{\operatorname{Div}}{|\Gamma|}\left\{\sum_{g \in \Gamma} f_{g}\left(\frac{1}{|\Gamma|} \sum_{\tilde{g} \in \Gamma} f_{\tilde{g} g^{\dagger} \tilde{g}^{\dagger}}\right) O_{g}^{I J} O_{g^{\dagger}}^{J I}\right. \\
\left.+\lambda \sum_{g \in \Gamma} f_{g} O_{g^{\dagger}}^{J I}\left[4 \delta^{I \hat{I}} \delta^{J \hat{J}}+\left(\delta^{I J}+R_{g}^{J I}\right) \delta^{\hat{I} \hat{J}}-2\left(\left(R_{g}^{-1}\right)^{I \hat{I}} \delta^{J \hat{J}}+\delta^{I \hat{I}}\left(R_{g}^{-1}\right)^{J \hat{J}}\right)\right] O_{g}^{\hat{I} \hat{J}}\right\} .
\end{gathered}
$$

One may recognize the bracket on the second line as the 1-loop dilatation operator acting on twisted operators.

- In [13] we considered a class of freely acting $\mathbb{Z}_{k}$ orbifolds possessing a global $S U(3)$ symmetry, where

$$
r\left(g_{n}\right)=\operatorname{diag}\left(\omega_{k}^{n}, \omega_{k}^{n}, \omega_{k}^{n}, \omega_{k}^{-3 n}\right), \quad \omega_{k}=e^{2 \pi i / k}
$$

and

$$
R\left(g_{n}\right)=\operatorname{diag}\left(\omega_{k}^{2 n}, \omega_{k}^{2 n}, \omega_{k}^{2 n}, \omega_{k}^{-2 n}, \omega_{k}^{-2 n}, \omega_{k}^{-2 n}\right),
$$

where $n=1, \ldots, k-1$ labels the twisted sector. There are two types of operators,

$$
O_{n}^{\langle i \bar{\jmath}\rangle}=\operatorname{Tr}\left(g_{n} \phi^{i} \phi^{\bar{\jmath}}\right)-\frac{1}{3} \eta^{i \bar{\jmath}} O_{n}, \quad O_{n}=\sum_{i=1}^{3} \operatorname{Tr}\left(g_{n} \phi^{i} \phi^{\bar{\imath}}\right)
$$

which form an octet and a singlet under the $S U(3)$, respectively. Their anomalous dimensions

$$
\gamma_{\mathbf{r}, n}=\frac{1}{16 \pi^{2} k} \delta_{\mathbf{r}, n}
$$

are found to be

$$
\delta_{\mathbf{8}, n}=8 \lambda \sin ^{2}(2 \pi x), \quad \delta_{\mathbf{1}, n}=2 \lambda[5+\cos (4 \pi x)]
$$


where

$$
x=\frac{n}{k} .
$$

Introducing the double-trace tree-level terms

$$
\delta S_{2 \text { trace }}^{\text {tree }}=\frac{1}{2} \sum_{n=1}^{k-1} f_{\mathbf{8}, n} O_{n}^{\langle i \bar{\jmath}\rangle} O_{-n}^{\langle j \bar{\imath}\rangle}+\frac{1}{2} \sum_{n=1}^{k-1} f_{\mathbf{1}, n} O_{n} O_{-n}
$$

with the symmetry

$$
f_{\mathbf{8}, n}=f_{\mathbf{8}, k-n} \quad f_{\mathbf{1}, n}=f_{\mathbf{1}, k-n}
$$

leads to the following beta functions:

$$
\begin{aligned}
& \beta_{8, n}=\frac{1}{32 \pi^{2} k}\left[\left(f_{8, n}+2 \delta_{8, n}\right)^{2}-256 \lambda^{2}(3+4 \cos (2 \pi x)) \sin ^{4}(\pi x)\right] \\
& \beta_{1, n}=\frac{3}{32 \pi^{2} k}\left[\left(f_{1, n}+\frac{2}{3} \delta_{1, n}\right)^{2}-\frac{64}{9} \lambda^{2}(1+2 \cos (2 \pi x))(4-2 \cos (2 \pi x)+\cos (4 \pi x))\right]
\end{aligned}
$$

The discriminants of both types of beta functions can become negative. The discriminant of the singlet beta function is negative for $1 / 3<x<2 / 3$; the negative region of the octet discriminant, $\cos (2 \pi x)<-3 / 4$, is completely included in this range.

\section{The role of $U(1)$ factors}

Let us consider an orbifold group $\Gamma$ of rank $k=|\Gamma|$. We start from the $\mathcal{N}=4 S U(k N)$ SYM and get $U(N)^{k} / U(1)=S U(N)^{k} \times U(1)^{k-1}$ as a result. Since the corresponding gauge group is not simple, we need to introduce the $U(1)$ coupling constant $e .{ }^{4}$ On the "natural line" [16] it is equal to the $S U(N)$ coupling constant $g$ at tree-level. Renormalization group flow drives $e$ to smaller values. There is no inheritance theorem for $e$ since $U(1)$ does not distinguish the single and double trace structures. Therefore, $\beta_{e}$ does not vanish; hence the orbifold theory is not conformal on the natural line. Far in the IR $e$ flows to zero and the $U(1)$ factors decouple.

Therefore, in order to search for a conformal theory we must remove the $U(1)$ factors and work with the $S U(N)^{k}$ gauge theory [13. Let us investigate how this modifies the beta-functions of other couplings. The background and quantum fields now must satisfy an additional constraint

$$
\operatorname{Tr}(g \Phi)=0 \quad(\forall) g \in \Gamma, \quad g \neq 1 .
$$

\footnotetext{
${ }^{4}$ The renormalization of all the $U(1)$ couplings is the same in a given orbifold theory since any node of the corresponding quiver diagram can be mapped into any other node by some orbifold group element.
} 
Therefore the propagator changes from $\left\langle\Phi_{\beta}^{\alpha} \Phi_{\delta}^{\gamma}\right\rangle=\frac{1}{2 N p^{2}} \delta_{\delta}^{\alpha} \delta_{\beta}^{\gamma}$ to

$$
\left\langle\Phi_{\beta}^{\alpha} \Phi_{\delta}^{\gamma}\right\rangle=\frac{1}{2 N p^{2}}\left[\delta_{\delta}^{\alpha} \delta_{\beta}^{\gamma}-\frac{1}{N} \delta_{\beta}^{\alpha} \delta_{\delta}^{\gamma}\right],
$$

where we suppressed possible space-time and flavor indices and did not take orbifold structure into account because it is not important for our consideration.

The new term $-\frac{1}{N} \delta_{\beta}^{\alpha} \delta_{\delta}^{\gamma}$ does not change the leading large $N$ one-loop result either for single-trace or for double-trace operators. ${ }^{5}$ Thus, the one-loop effective potential and the beta-functions are not modified to leading order in $1 / N$ by removing the $U(1)$ factors. The only modification appears at tree level; the $U(N)$ potential

$$
\lambda N \operatorname{Tr}\left|\left[\Phi_{\mu}, \Phi_{\nu}\right]\right|^{2}=\lambda N\left[\operatorname{Tr}\left|\sum_{i}\left[\Phi_{i}, \Phi_{i}^{\dagger}\right]\right|^{2}+4 \operatorname{Tr} \sum_{i \neq j}\left|\left[\Phi_{i}, \Phi_{j}\right]\right|^{2}\right]
$$

is corrected by the addition of the double-trace terms

$$
-\lambda \sum_{g \in \Gamma}\left[\left|\sum_{i} \operatorname{Tr} g\left[\Phi_{i}, \Phi_{i}^{\dagger}\right]\right|^{2}+4 \sum_{i \neq j}\left|\operatorname{Tr} g\left[\Phi_{i}, \Phi_{j}\right]\right|^{2}\right]
$$

which arise from integrating out the twisted $U(1)$ auxiliary fields in the vector and chiral superfields, respectively. Here $\Phi_{i}, i=1,2,3$, are the complex scalar fields.

We conclude that the only difference between the $S U(N)^{k}$ and $U(N)^{k} / U(1)$ is the double trace operator that appears at the tree level. Let us clarify the relation of this fact to our previous work [13] where the one-loop renormalizability required us to deform the theory by double-trace operators. Now we note that such operators are present already at tree level in the $S U(N)^{k}$ gauge theories. We will now show that, in all supersymmetric orbifolds, the tree-level coefficients (23) are in fact the RG fixed points of the double-trace couplings.

\section{1 $\mathcal{N}=2$ supersymmetric orbifolds}

Let us illustrate the discussion above by considering $\mathcal{N}=2$ supersymmetric $\mathbb{Z}_{k}$ orbifold theories. We define the action of $\mathbb{Z}_{k}$ by

$$
r=\left(\begin{array}{cccc}
e^{i m \alpha} & 0 & 0 & 0 \\
0 & e^{-i m \alpha} & 0 & 0 \\
0 & 0 & 1 & 0 \\
0 & 0 & 0 & 1
\end{array}\right) \quad \alpha=\frac{2 \pi}{k}
$$

\footnotetext{
${ }^{5}$ We expect this to be true also for the higher loops, but have not checked them explicitly.
} 
The scalar field content of the quiver gauge theory is the following: a complex (adjoint) field $\Phi=\Phi_{3}$ and "bi-fundamental" fields $Q=\Phi_{2}$ and $\tilde{Q}=\Phi_{1}$ which form an $\mathcal{N}=2$ hypermultiplet. These fields are eigenvectors for $\mathbb{Z}_{k} \in S O(6)$

$$
\begin{aligned}
& R(\Phi)=\Phi \quad R\left(\Phi^{\dagger}\right)=\Phi^{\dagger} \\
& R(Q)=Q e^{i m \alpha} \quad R\left(Q^{\dagger}\right)=Q^{\dagger} e^{-i m \alpha} . \\
& R(\tilde{Q})=\tilde{Q} e^{-i m \alpha} \quad R(\tilde{Q})^{\dagger}=\tilde{Q}^{\dagger} e^{+i m \alpha}
\end{aligned}
$$

The divergent part of the double-trace contribution to the one-loop effective action follows from our general formulae:

$$
\begin{aligned}
& \delta S^{1 \text { loop } \mid 2 \operatorname{tr}}=-\frac{\lambda^{2} \operatorname{Div}}{2 k} \sum_{n=1}^{k-1} 8(1-\cos (n m \alpha))^{2}\left[\operatorname{Tr}\left(g_{n} Q Q^{\dagger}\right) \operatorname{Tr}\left(g_{n}^{\dagger} Q Q^{\dagger}\right)\right. \\
& \left.\quad+\operatorname{Tr}\left(g_{n} \tilde{Q} \tilde{Q}^{\dagger}\right) \operatorname{Tr}\left(g_{n}^{\dagger} \tilde{Q} \tilde{Q}^{\dagger}\right)-2 \operatorname{Tr}\left(g_{n} Q Q^{\dagger}\right) \operatorname{Tr}\left(g_{n}^{\dagger} \tilde{Q} \tilde{Q}^{\dagger}\right)+4 \operatorname{Tr}\left(g_{n} Q \tilde{Q}\right) \operatorname{Tr}\left(g_{n}^{\dagger} \tilde{Q}^{\dagger} Q^{\dagger}\right)\right] \quad(26) \\
& =-\frac{\lambda^{2} \operatorname{Div}}{2 k} \sum_{n=1}^{k-1} 4(1-\cos (n m \alpha))\left[\left|\operatorname{Tr}\left(g_{n}\left[Q, Q^{\dagger}\right]\right)-\operatorname{Tr}\left(g_{n}\left[\tilde{Q}^{\dagger}, \tilde{Q}\right]\right)\right|^{2}+4\left|\operatorname{Tr}\left(g_{n}[Q, \tilde{Q}]\right)\right|^{2}\right]
\end{aligned}
$$

Note that the adjoint field $\Phi$ does not appear in the answer.

In the previous section we observed that the tree-level potential of the $S U(N)^{k}$ quiver gauge theory, i.e. the orbifold theory with the $U(1)$ factors removed, contains the doubletrace operators

$$
\frac{1}{k} \sum_{n=1}^{k-1} f_{n}\left[\left|\operatorname{Tr}\left(g_{n}\left[Q, Q^{\dagger}\right]\right)+\operatorname{Tr}\left(g_{n}\left[\tilde{Q}^{\dagger}, \tilde{Q}\right]\right)\right|^{2}+4\left|\operatorname{Tr}\left(g_{n}[Q, \tilde{Q}]\right)\right|^{2}\right]
$$

with coupling constants $f_{n}=-\lambda$. We will now check that precisely for this value the double-trace beta function vanishes. Indeed, we find

$$
\beta_{f_{n}}=4 \frac{(1-\cos (n m \alpha))}{16 \pi^{2} k}\left(\lambda+f_{n}\right)^{2}
$$

Therefore, as expected, there is a fixed line $f_{n}=-\lambda$ corresponding to the $\mathcal{N}=2$ superconformal $S U(N)^{k}$ gauge theory.

Let us remark that, as a consequence of the choice of the orbifold action (24), the $S U(2)_{R}$ symmetry of the orbifold theory is not a subgroup of the $S U(3)$ manifest in $\mathcal{N}=1$ superspace. Indeed, the $S U(2) \subset S U(3)$ leaving $\Phi$ invariant and rotating

$$
\left(\begin{array}{l}
Q \\
\tilde{Q}
\end{array}\right)
$$


as a doublet does not commute with the orbifold group (25). The $S U(2)_{R^{-}}$doublets are rather

$$
q_{1}^{i}=\left(\begin{array}{c}
Q \\
\tilde{Q}^{\dagger}
\end{array}\right) \quad q_{2}^{i}=\epsilon_{i j}\left(q_{1}^{j}\right)^{*}=\left(\begin{array}{c}
\tilde{Q} \\
-Q^{\dagger}
\end{array}\right)
$$

It is straightforward to check that (26) is invariant under $S U(2)$ transformations of (30), being a particular case of the following manifestly invariant expression:

$$
\begin{aligned}
& \operatorname{Tr}\left(g_{n} q_{1}^{i} q_{2}^{j}\right) \operatorname{Tr}\left(g_{n}^{\dagger} q_{1}^{k} q_{2}^{l}\right)\left(B \epsilon_{i k} \epsilon_{j l}-A \epsilon_{i l} \epsilon_{j k}\right)=2(A+B) \operatorname{Tr}\left(g_{n} Q Q^{\dagger}\right) \operatorname{Tr}\left(g_{n}^{\dagger} \tilde{Q}^{\dagger} \tilde{Q}\right) \\
& \quad+A\left(\operatorname{Tr}\left(g_{n} Q Q^{\dagger}\right) \operatorname{Tr}\left(g_{n}^{\dagger} Q Q^{\dagger}\right)+\operatorname{Tr}\left(g_{n} \tilde{Q} \tilde{Q}^{\dagger}\right) \operatorname{Tr}\left(g_{n}^{\dagger} \tilde{Q} \tilde{Q}^{\dagger}\right)\right)-2 B \operatorname{Tr}\left(g_{n} Q \tilde{Q}\right) \operatorname{Tr}\left(g_{n}^{\dagger} \tilde{Q}^{\dagger} Q^{\dagger}\right)
\end{aligned}
$$

\section{$3.2 \mathcal{N}=1$ supersymmetric orbifolds}

It is not hard to perform a similar analysis for the $\mathcal{N}=1$ supersymmetric $\mathbb{Z}_{k}$ orbifolds. The action of the generator in the fundamental representation of the R-symmetry group is

$$
r=\left(\begin{array}{cccc}
e^{i m_{1} \alpha} & 0 & 0 & 0 \\
0 & e^{i m_{2} \alpha} & 0 & 0 \\
0 & 0 & e^{-i\left(m_{1}+m_{2}\right) \alpha} & 0 \\
0 & 0 & 0 & 1
\end{array}\right) \quad \alpha=\frac{2 \pi}{k}
$$

with the action on the three complex scalar fields $\Phi_{i}$ defined in the usual way. This action can be easily generalized to arbitrary abelian orbifolds.

The double-trace terms induced at one-loop level are

$\delta S^{1 \text { loop } \mid 2 \operatorname{tr}}=\frac{\lambda^{2} \operatorname{Div}}{k} \sum_{n=1}^{k-1}\left[3-\cos \left(n m_{1} \alpha\right)-\cos \left(n m_{2} \alpha\right)-\cos \left(m_{1}+m_{2}\right) n \alpha\right]\left|\sum_{i} \operatorname{Tr}\left(g_{n}\left[\Phi_{i}, \Phi_{\bar{\imath}}\right]\right)\right|^{2}$,

where $g_{n}$ is the $n$-th element of $\mathbb{Z}_{k}$. The tree-level potential of the $S U(N)^{k}$ theory contains

$$
\frac{1}{k} \sum_{n=1}^{k-1} f_{n}\left|\sum_{i} \operatorname{Tr}\left(g_{n}\left[\Phi_{i}, \Phi_{\bar{\imath}}\right]\right)\right|^{2}
$$

with $f_{n}=-\lambda$. The beta-function for $f_{n}$ is found to be

$$
\beta_{f_{n}}=\frac{1}{8 \pi^{2} k}\left[3-\cos \left(n m_{1} \alpha\right)-\cos \left(n m_{2} \alpha\right)-\cos \left(m_{1}+m_{2}\right) n \alpha\right]\left(\lambda+f_{n}\right)^{2},
$$

which indeed vanishes for this value of the coupling. Therefore, as expected, there is a fixed line of $\mathcal{N}=1$ superconformal $S U(N)^{k}$ gauge theories. 
This structure of $\beta_{f_{n}}$ has the following explanation in the $S U(N)^{k} \times U(1)^{k-1}$ gauge theory. Since $\lambda+f_{n} \sim e^{2} N$, where $e$ is the coupling of the $U(1)$ factors, (35) may be rewritten as

$$
\beta_{e} \sim e^{3} N
$$

i.e., the double-trace beta function is related by supersymmetry to the beta function for the $U(1)$ charge. Thus, the supersymmetry of the $S U(N)^{k} \times U(1)^{k-1}$ gauge theory explains the universal $\left(\lambda+f_{g}\right)^{2}$ form of the double-trace beta function. It follows that the beta functions for all double-trace operators generated at one-loop level have vanishing discriminants $D$ in all supersymmetric orbifold gauge theories. This fact will be important in comparing the field theory results with closed string theory.

In the next section we will discuss general abelian orbifold theories of which the $\mathbb{Z}_{k}$ orbifolds discussed here are a special case. The coupling constants $f_{n}$ in equation (34) acquire an additional label $f_{n} \rightarrow f_{n, i}$, and the existence of a fixed line will be tested through the signs of the eigenvalues of a matrix discriminant. In comparing this more general setup with the present discussion, we will encounter cases where

$$
\beta_{f_{O}}=f_{O}^{2}+2 \lambda \gamma_{O} f_{O}
$$

i.e. the operator is not induced by one-loop diagrams. In these cases $D=\gamma_{O}^{2}>0$, and there are two solutions, $f_{O}=0$ and $f_{O}=-2 \gamma_{O} \lambda$. Taking $\gamma_{O}>0$ we note that only $f_{O}=0$ is an IR stable fixed point. Hence, we pick this solution which preserves supersymmetry.

\section{General abelian orbifolds}

Now we are ready to consider general abelian orbifolds. All group elements can be simultaneously brought to a diagonal form

$$
r=\left(\begin{array}{cccc}
e^{2 \pi i x_{1}} & 0 & 0 & 0 \\
0 & e^{2 \pi i x_{2}} & 0 & 0 \\
0 & 0 & e^{2 \pi i x_{3}} & 0 \\
0 & 0 & 0 & e^{-2 \pi i\left(x_{1}+x_{2}+x_{3}\right)}
\end{array}\right)
$$

perhaps with an additional set of integer labels. For example, if $\Gamma=\mathbb{Z}_{k_{1}} \times \cdots \times \mathbb{Z}_{k_{s}}$, the exponents $x_{i}$ are given by

$$
x_{i}=\sum_{j=1}^{s} m_{i j} \frac{n_{j}}{k_{j}},
$$

where $m_{i j}$ is a set of integers labeling the group representation, and $n_{j}$ label the group elements. For ease of notation we further introduce the variables

$$
y_{i}=2 \pi x_{i}, \quad y=y_{1}+y_{2}+y_{3}
$$


We will show that there is no fixed line unless the theory is supersymmetric, which happens either when one of the parameters $x_{i}$ is an integer, or when $x_{1}+x_{2}+x_{3}$ is an integer.

To reach this conclusion it is sufficient to analyze the operators $\operatorname{Tr}\left(g \Phi_{i} \Phi_{\bar{\imath}}\right) \operatorname{Tr}\left(g^{\dagger} \Phi_{j} \Phi_{\bar{\jmath}}\right)$ where no summation over repeated indices is implied. Such operators can be constructed from the spectrum of general abelian orbifolds. In cases in which some nonabelian global symmetry survives the orbifold projection, some of the single-trace factors of the doubletrace operators belong to nontrivial representations of this global symmetry group, such as $\operatorname{Tr}\left(g \Phi_{i} \Phi_{\bar{\jmath}}\right) \operatorname{Tr}\left(g^{\dagger} \Phi_{j} \Phi_{\bar{\imath}}\right)$. The surviving nonabelian global symmetry prevents them from mixing with $\left|\operatorname{Tr}\left(g \Phi_{i} \Phi_{j}\right)\right|^{2}$ when the latter exist. Thus, the operators $\operatorname{Tr}\left(g \Phi_{i} \Phi_{\bar{\imath}}\right) \operatorname{Tr}\left(g^{\dagger} \Phi_{j} \Phi_{\bar{\jmath}}\right)$ are a universal guide to the behaviour of orbifold theories.

For further convenience we introduce the notation

$$
O_{i}^{g}=\frac{1}{\cos \frac{1}{2}\left(y-y_{i}\right)} \operatorname{Tr}\left(g\left\{\Phi_{i}, \Phi_{\bar{\imath}}\right\}\right)=\frac{1}{\cos \frac{1}{2}\left(y-y_{i}\right)} \operatorname{Tr}\left(g \Phi_{i} \Phi_{\bar{\imath}}+g \Phi_{\bar{\imath}} \Phi_{i}\right) .
$$

The prefator in the definition above is necessary to make the operator $O_{i}^{g}$ well-defined for the orbifold group elements which anticommute with at least one of the scalar fields.

In terms of $O_{i}^{g}$ the 1-loop effective potential could be written as

$$
\delta S^{1 \text { loop } \mid 2 \operatorname{tr}}=\frac{\lambda^{2} \mathrm{Div}}{16 \pi^{2} k} \sum_{g} \sum_{i, j} \cos \frac{1}{2}\left(y-y_{i}\right) \cos \frac{1}{2}\left(y-y_{j}\right) h_{i j}^{g} O_{i}^{g} O_{j}^{g^{\dagger}} .
$$

The reality of the effective action requires that the matrix $h_{i j}^{g}$ be hermitian $\left(h_{i j}^{g}\right)^{*}=h_{i j}^{g^{\dagger}}=h_{j i}^{g}$; It turns out however that it is actually real and symmetric. All its elements can be obtained by a suitable relabeling of the entries $h_{11}^{g}$ and $h_{12}^{g}$ :

$$
\begin{aligned}
& \cos ^{2}\left(\frac{y_{2}+y_{3}}{2}\right) h_{11}^{g}=-8 \cos \left(\frac{y_{1}+y_{2}}{2}\right) \cos \left(\frac{y_{2}+y_{3}}{2}\right) \cos \left(\frac{y_{3}+y_{1}}{2}\right)+2\left(1-\cos \left(y_{2}+y_{3}\right)\right) \\
&+\left(1+\cos ^{2}\left(\frac{y_{2}+y_{3}}{2}\right)\right)\left(1+\cos \left(y_{1}+y_{2}\right)+\cos \left(y_{2}+y_{3}\right)+\cos \left(y_{3}+y_{1}\right)\right) \\
& \cos \left(\frac{y_{2}+y_{3}}{2}\right) \cos \left(\frac{y_{1}+y_{3}}{2}\right) h_{12}^{g}=-4 \cos \left(\frac{y_{1}+y_{2}}{2}\right) \\
&+\cos \left(\frac{y_{2}+y_{3}}{2}\right) \cos \left(\frac{y_{1}+y_{3}}{2}\right)\left[5+\cos \left(y_{1}+y_{2}\right)-\cos \left(y_{2}+y_{3}\right)-\cos \left(y_{1}+y_{3}\right)\right]
\end{aligned}
$$

The effective action (42) implies that the deformation (8) involves a hermitian matrix of tree-level couplings $f_{i j}^{g}$

$$
\delta S_{\text {tree }}=\frac{1}{2 k} \sum_{g} f_{i j}^{g} O_{i}^{g} O_{j}^{g^{\dagger}}
$$


The explicit form of the corresponding beta-function is rather involved,

$$
\begin{aligned}
\beta_{f_{i j}^{g}}= & \frac{1}{16 \pi^{2} k}\left(2 \lambda^{2}\left[\cos \frac{1}{2}\left(y-y_{i}\right) \cos \frac{1}{2}\left(y-y_{j}\right) h_{i j}^{g}\right]+2 \lambda f_{i j}^{g}\left(2-\cos \left(y-y_{i}\right)-\cos \left(y-y_{j}\right)\right)\right. \\
& \left.+2 \lambda f_{i k}^{g} \cos \left(\frac{y-y_{k}}{2}\right) \cos \left(\frac{y-y_{j}}{2}\right)+2 \lambda f_{k j}^{g} \cos \left(\frac{y-y_{i}}{2}\right) \cos \left(\frac{y-y_{k}}{2}\right)+f_{i k}^{g} f_{k j}^{g}\right),
\end{aligned}
$$

but can be rearranged in a convenient form

$$
\tilde{\beta}_{f_{i j}^{g}}=\left(\tilde{f}_{i k}^{g}+A_{i k}^{g}\right)\left(\tilde{f}_{k j}^{g}+A_{k j}^{g}\right)-4 \lambda^{2} M_{i j}^{g}
$$

exposing the matrix analog $M_{i j}^{g}$ of the discriminant $D$. Here

$$
A_{i j}^{g}=2 \lambda\left(\delta_{i j}\left(1-\cos \left(y-y_{i}\right)\right)+\cos \left(\frac{y-y_{i}}{2}\right) \cos \left(\frac{y-y_{j}}{2}\right)\right),
$$

and the explicit form of the hermitian matrix $M_{i j}^{g}$ is

$$
\begin{aligned}
M_{i j}^{g} & =-\frac{1}{2} \cos \left(\frac{y-y_{i}}{2}\right) \cos \left(\frac{y-y_{j}}{2}\right) h_{i j}^{g}+\frac{1}{4 \lambda^{2}} A_{i k}^{g} A_{k j}^{g}, \\
M_{i i}^{g} & =1-\frac{1}{2} \cos \left(y-y_{i}\right)-\frac{1}{2} \sum_{k=1}^{3} \cos \left(y-y_{k}\right)+4 \prod_{k=1}^{3} \cos \left(\frac{y-y_{k}}{2}\right), \\
M_{i j}^{g} & =\cos \left(\frac{y-y_{i}}{2}\right) \cos \left(\frac{y-y_{j}}{2}\right)+\left.2 \cos \left(\frac{y_{i}+y_{j}}{2}\right)\right|_{i \neq j} .
\end{aligned}
$$

This matrix is obviously invariant under $y_{i} \rightarrow 2 \pi-y_{i}$ for all $i=1,2,3$ : this is a manifestation of the symmetry under $g \rightarrow g^{-1}$. Another symmetry is related to the choice of basis on the maximal torus of $S U(4)$. The change $y_{3} \rightarrow-y_{1}+y_{2}+y_{3}$ combined with interchange of indices 1 and $2(1 \leftrightarrow 2)$ leaves the matrix $M_{i j}$ invariant.

If we can find an orbifold group element $g$ such that at least one eigenvalue of $M$ is negative, then at least one beta function does not vanish for any real value of the corresponding tree-level coupling and consequently conformal invariance is broken. Therefore, the smallest eigenvalue of $M_{i j}$ as a function of $x_{i}$ is a useful quantity which controls the existence of a fixed line.

Now we find the region inside the unit cube $0 \leq x_{i}<1$, i.e. $0 \leq y_{i}<2 \pi$, where the smallest eigenvalue is negative. Let us start from the points where supersymmetry is preserved. They are the edges of the cube $x_{i}=0$ or the planes $x_{1}+x_{2}+x_{3}=1,2$. The fixed line exists in these cases, as was shown in section 3. For example, if $y_{3}=-y_{1}-y_{2}$ we have

$$
\begin{aligned}
M_{i j} & =\frac{1}{4 \lambda^{2}}(\tilde{f}+A)^{2}, \\
\frac{1}{2 \lambda}(\tilde{f}+A) & =\left(\begin{array}{ccc}
1 & \cos \left(y_{3}+\frac{y_{1}+y_{2}}{2}\right) & \cos \left(y_{2}+\frac{y_{1}+y_{3}}{2}\right) \\
\cos \left(y_{3}+\frac{y_{1}+y_{2}}{2}\right) & 1 & \cos \left(y_{1}+\frac{y_{2}+y_{3}}{2}\right) \\
\cos \left(y_{2}+\frac{y_{1}+y_{3}}{2}\right) & \cos \left(y_{1}+\frac{y_{2}+y_{3}}{2}\right) & 1
\end{array}\right) \\
y_{3} & =-y_{1}-y_{2}
\end{aligned}
$$


and therefore for any choice of $y_{i}$ its smallest eigenvalue is equal to zero. ${ }^{6}$

A further analysis shows that these are the only hypersurfaces where the smallest eigenvalue of $M_{i j}$ is equal to zero. As a result, the cube $0 \leq x_{i}<1$ is divided into three regions: $0 \leq x_{1}+x_{2}+x_{3} \leq 1,1<x_{1}+x_{2}+x_{3}<2$ and $2 \leq x_{1}+x_{2}+x_{3} \leq 3$. In the first and the third regions, which are related by the $x_{i} \rightarrow 1-x_{i}$ symmetry, all eigenvalues of $M$ are non-negative. In the "middle" region $1<x_{1}+x_{2}+x_{3}<2$ at least one eigenvalue of $M$ is negative: this is the unstable region. This structure is shown in Figure 1.

\section{Closed String Zero-Point Energy}

To calculate the spectrum of twisted closed strings on $\mathbb{R}^{3,1} \times \mathbb{R}^{6} / \Gamma$, it is efficient to use the light-cone gauge Green-Schwarz methods. We refer, e.g., to [17] for an application to a simple $\mathbb{Z}_{4}$ orbifold which we now generalize to arbitrary abelian orbifolds.

Let us consider first $\Gamma=\mathbb{Z}_{k}$. Then, the equation (38) gives the action of $\mathbb{Z}_{k}$ in the fundamental representation of $S U(4)$, while the action in the vector representation of $S O(6)$ is

$$
R\left(g_{n}\right)=\operatorname{diag}\left(\omega_{k}^{n\left(m_{1}+m_{2}\right)}, \omega_{k}^{n\left(m_{1}+m_{3}\right)}, \omega_{k}^{n\left(m_{2}+m_{3}\right)}, \omega_{k}^{-n\left(m_{1}+m_{2}\right)}, \omega_{k}^{-n\left(m_{1}+m_{3}\right)}, \omega_{k}^{-n\left(m_{2}+m_{3}\right)}\right),
$$

where $\omega_{k}=e^{2 \pi i / k}$.

In the light-cone Green-Schwarz approach one finds four complex world sheet bosonic coordinates $X^{l}$ and four complex fermionic coordinates $b^{l}$. In the $n$-twisted sector we find that the fermionic cordinates have boundary conditions

$$
b^{l}(\sigma+2 \pi)=e^{2 \pi i n m_{l} / k} b^{l}(\sigma)
$$

and the bosonic coordinates

$$
\begin{aligned}
& X^{1}(\sigma+2 \pi)=X^{1}(\sigma), \\
& X^{2}(\sigma+2 \pi)=e^{2 \pi i n\left(m_{1}+m_{2}\right) / k} X^{2}(\sigma), \\
& X^{3}(\sigma+2 \pi)=e^{2 \pi i n\left(m_{1}+m_{3}\right) / k} X^{3}(\sigma), \\
& X^{4}(\sigma+2 \pi)=e^{2 \pi i n\left(m_{2}+m_{3}\right) / k} X^{4}(\sigma) .
\end{aligned}
$$

These equations can be trivially extended to general abelian orbifolds (38) by making the replacements $n m_{l} / k \rightarrow x_{l}$, etc. ${ }^{7}$

${ }^{6} \frac{1}{2 \lambda}(\tilde{f}+A)$ has the form (53) only for $y_{3}=-y_{1}-y_{2}$. Changing the choice of supersymmetry constraint requires appropriate reshuffling of $y_{1,2,3}$ in (53).

${ }^{7}$ It is worth pointing out that the equation (38) covers all possible orbifold actions. Similarly to the $\mathbb{Z}_{4}$ 
The zero-point energy due to a complex boson with boundary condition $X(\sigma+2 \pi)=$ $e^{2 \pi i \theta} X(\sigma)$ is

$$
\frac{1}{24}-\frac{(2\{\theta\}-1)^{2}}{8}
$$

where $\{\theta\}$ is the fractional part of $\theta$, which ranges from 0 to 1 . For a fermion we get the opposite of this quantity. Using the variables $x_{i}, i=1,2,3$ introduced in the previous section, we find that the energy of the ground state is given by

$$
\begin{aligned}
8 E_{0}\left(x_{1}, x_{2}, x_{3}\right)= & -1-\left(2\left\{x_{1}+x_{2}\right\}-1\right)^{2}-\left(2\left\{x_{1}+x_{3}\right\}-1\right)^{2}-\left(2\left\{x_{2}+x_{3}\right\}-1\right)^{2} \\
& +\left(2\left\{x_{1}+x_{2}+x_{3}\right\}-1\right)^{2}+\sum_{i=1}^{3}\left(2\left\{x_{i}\right\}-1\right)^{2}
\end{aligned}
$$

Since $E_{0}\left(x_{1}, x_{2}, x_{3}\right)$ is periodic in each of the variables with period 1 , it is sufficient to determine it inside the unit cube $0 \leq x_{i}<1$. Just as the matrix $M_{i j}, E_{0}$ also has a discrete symmetry

$$
E_{0}\left(1-x_{1}, 1-x_{2}, 1-x_{3}\right)=E_{0}\left(x_{1}, x_{2}, x_{3}\right) .
$$

Let us divide the unit cube into three regions by the planes $x_{1}+x_{2}+x_{3}=1$ and $x_{1}+x_{2}+x_{3}=2$, as in Figure 1. In the lower region $0 \leq x_{1}+x_{2}+x_{3} \leq 1$, we also have $0 \leq x_{i}+x_{j} \leq 1$, so that all \{\} symbols may be removed from (59). Then it is easy to see that $E_{0}$ vanishes identically in the lower region. By the symmetry (601) this is also true in the upper region.

In the region between the planes, $1<x_{1}+x_{2}+x_{3}<2$, the function has a different form depending on whether $x_{i}+x_{j}$ exceeds unity, but it is not hard to see that $E_{0}$ is negative in the entire middle region. Thus, only the middle region corresponds to a tachyonic instability.

\section{Comparison}

In the last two sections we showed that the region of instability of the one-loop orbifold gauge theory coincides with that of the free closed superstrings on $\mathbb{R}^{3,1} \times \mathbb{R}^{6} / \Gamma$. This is summarized in Figure 1, the unstable region being contained between the two shaded planes. The three axes correspond to the $x_{i}$ with $i=1,2,3$. A point in this region corresponds both to a gauge theory operator with nonvanising beta function as well as to a closed string tachyonic

case discussed in [17], orbifolds for which the values $x_{i}=1 / 2$ are allowed can be thought of as orbifolds of the type 0 theory rather than of the type $I I$ theory. One may translate them to the type II theory by multiplying the fermion action by $(-)^{\mathbf{F}}\left[18\right.$. This simply amounts to the redefinition $x_{i} \rightarrow x_{i}+1 / 2$ which leads to a reshufling of the fermion charges. This shift is inconsequential for the orbifold action in the vector representation. 


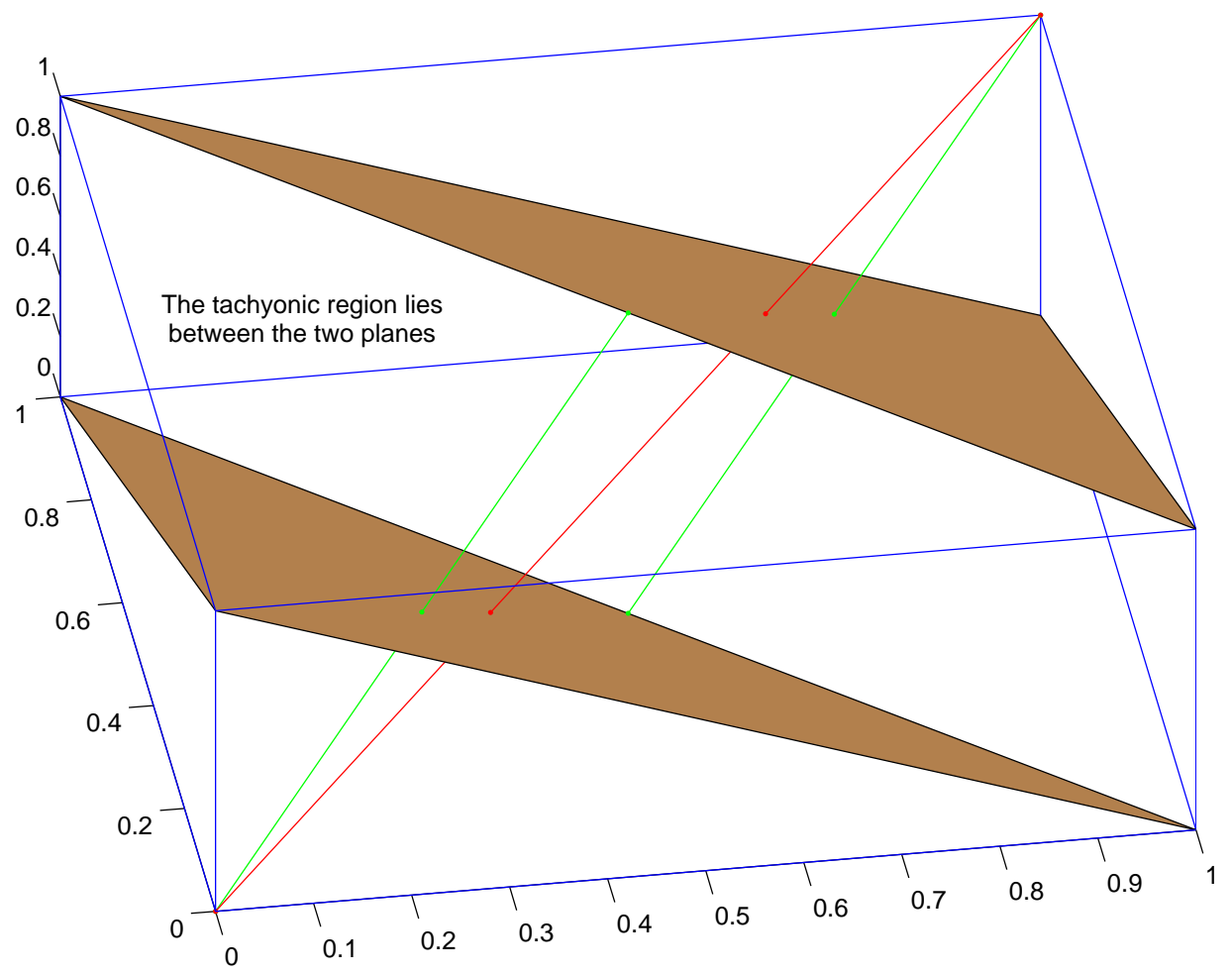

Figure 1: Stability/instability regions for general abelian orbifolds

ground state. Here we further discuss this surprising correspondence and illustrate it with some simple examples.

We will start with supersymmetric theories. The orbifold preserves supersymmetry if and only if all the double-trace operators correspond to points on the hypersurfaces $x_{1}+$ $x_{2}+x_{3}=0 \bmod 1$. In these cases the string zero-point energy is equal to zero and also equal to the smallest eigenvalue of the matrix $M$. A simple example of such a theory is the $S U(3)$ symmetric $\mathbb{Z}_{3}$ orbifold. As discussed in [13], this theory has an untwisted sector, $n=0, x_{i}=0$, and two twisted sectors, $n=1, x_{i}=1 / 3$ and $n=2, x_{i}=2 / 3$. All these points (red dots) lie on the "supersymmetric" hypersurfaces $x_{1}+x_{2}+x_{3}=0 \bmod 1$ as shown in Figure 1].

Now let us discuss the class of $\mathbb{Z}_{k}$ orbifolds with $S U(3)$ global symmetry. This class of theories was discussed extensively in [13 and corresponds to $m_{1}=m_{2}=m_{3}$, i.e. to moving along the line $x_{1}=x_{2}=x_{3}=x$ which is shown in red in Figure 1 . In this case $E_{0}=0$ for $0 \leq x \leq 1 / 3$, and

$$
E_{0}(x)=1-3 x, \quad 1 / 3 \leq x \leq 1 / 2 .
$$

Thus, the twisted sector ground states with small charges, $n<k / 3$, are not tachyonic, but 
for $k / 3<n<2 k / 3$ they are. Comparing this with the plot of the singlet discriminant $D$ in the field theory calculation of [13], we observe a remarkable agreement: a transition from stability to instability at $x=1 / 3$ and back to stability at $x=2 / 3$. This result was already derived in [13], but let us trace here how it appears from the matrix (49). In this case $M_{i j}$ may be simplified to

$$
M_{i i}=-4 \cos ^{2}(2 \pi x)+3+4 \cos ^{3}(2 \pi x), \quad M_{i \neq j}=\cos (2 \pi x)(\cos (2 \pi x)+2) .
$$

The eigenvalue which becomes negative first as $x$ is increased,

$$
(1+2 \cos (2 \pi x))(4-2 \cos (2 \pi x)+\cos (4 \pi x)),
$$

coincides up to an irrelevant numerical factor with the discriminant for the singlet betafunction (cf. equation (19)). Its sign changes from positive to negative values at $x=1 / 3$, and back to positive at $x=2 / 3$, in perfect agreement with the closed string zero-point energy (cf. the solid line portions of the two curves in Figure 2).

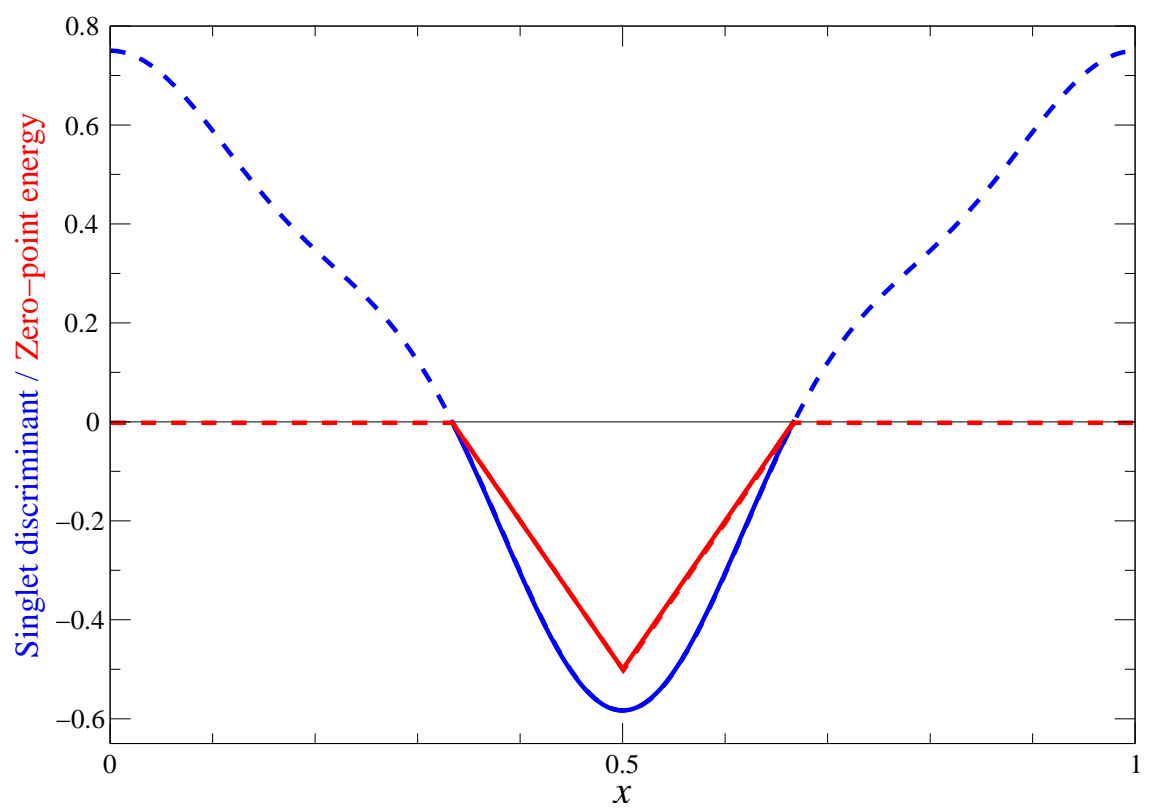

Figure 2: The (rescaled) singlet discriminant and the closed string zero-point energy. Both are negative in the same range of $x$.

The other two eigenvalues coincide and are proportional to the discriminant of the octet 
beta function (18): ${ }^{8}$

$$
(3+4 \cos (2 \pi x))(1+\cos (2 \pi x))^{2},
$$

which crosses from positive to negative values at $\cos (2 \pi x)=-3 / 4$, i.e. at $x \approx 0.385$. Since this is greater than $1 / 3$, the octet instability indeed sets in after the initial singlet instability. We suggest that the octet instability is associated with an $S U(3)$ octet excited closed string state becoming tachyonic. Let us consider the region $1 / 3<x<1 / 2$ where the ground state energy is $(1-3 x)$. For each of the three twisted complex worldsheet fields $X^{i}$, the lowest oscillator excitation energy in this case is $(1-2 x)$. So, the excited closed strings obtained by acting with one of these oscillators on the left and one on the right,

$$
\alpha_{2 x-1}^{i} \tilde{\alpha}_{2 x-1}^{\bar{j}}|0\rangle
$$

are tachyonic for $2-5 x<0$, i.e. for $x>2 / 5$. Numerically, this is close to the gauge theory value $x \approx 0.385$. Perhaps the lack of exact agreement can be attributed to the fact that the singlet tachyon is already condensed, which has not been taken into account in our calculations.

Another simple example, which has $S U(2)$ symmetry, is $m_{3}=2 m_{2}=2 m_{1}$. This corresponds to the line $x_{1}=x_{2}=x, x_{3}=2 x$, which is shown in blue in Figure 1 . In this case the transition from stability to instability takes place at $x=1 / 4$, and $x=1 / 2$ is an isolated supersymmetric point that is stable. This behavior is shown in the Figure 3.

The first eivenvalue of the matrix $M$ to become negative is $(z=\cos (2 \pi x))$

$$
\mathcal{M}_{1}^{d}=\frac{1}{2}\left(4+z\left(12-15 z-8 z^{2}+16 z^{3}\right)-(2+z) \sqrt{4-12 z+z^{2}+16 z^{3}}\right) \quad ;
$$

it is negative for $1 / 4<x<1 / 2$, in agreement with the behavior of the zero-point energy. The ranges of $x$ for which the other two eigenvalues become negative

$$
\begin{aligned}
& \mathcal{M}_{2}^{d}=\frac{1}{2}\left(4+z\left(12-15 z-8 z^{2}+16 z^{3}\right)+(2+z) \sqrt{4-12 z+z^{2}+16 z^{3}}\right) \\
& \mathcal{M}_{3}^{d}=(1-z)^{2}\left(1+8 z+8 z^{2}\right)
\end{aligned}
$$

are completely contained in $1 / 4<x<1 / 2$. This suggests that the subleading instabilities associated with the higher eigenvalues crossing zero are again dual to excited closed strings becoming tachyonic. This would be an interesting comparison to pursue in the future.

Generically, the eigenvalues of the disciminant matrix cross as functions of the twisted sector charge. This effect is in fact crucial for the agreement of the gauge theory ans string theory instabilities. An example corresponding to the $S U(2)$-symmetric orbifold with weights $5 m_{1}=m_{2}=m_{3}$ is shown in Figure 4. It is easy to identify on the figure the points where

${ }^{8}$ Although we reproduce the result of [13, note that here we discuss only the "diagonal" operators $\operatorname{Tr}\left(g \Phi_{i} \Phi_{\bar{\imath}}\right)$ while in [13] more general operators were considered. As discussed at the beginning of secton 4, the apparently missing operators can be obtained from the ones we considered here through the action of $S U(3)$, which for this choice of orbifold survives as nonabelian global symmetry. 


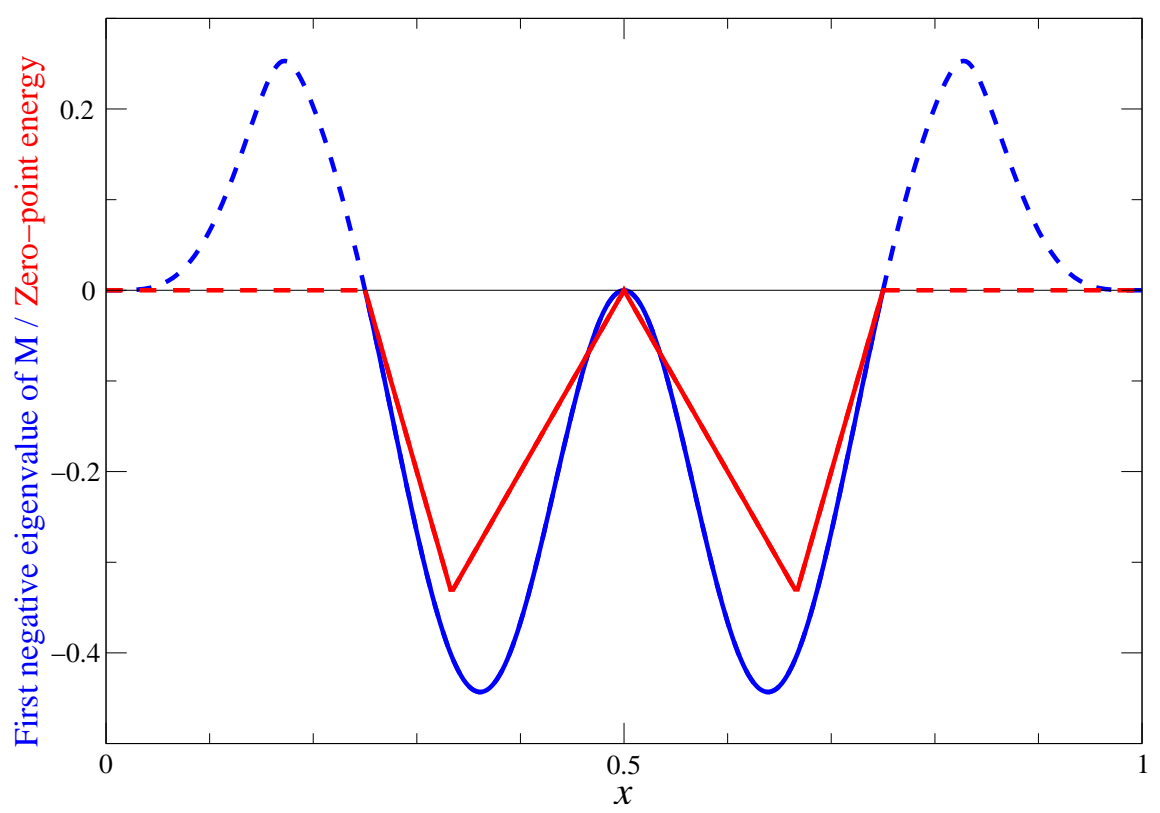

Figure 3: The (rescaled) eigenvalue $\mathcal{M}_{1}^{d}$ and the closed string zero-point energy, plotted as a function of $x$. Both are negative (the solid line portions of the two curves) in the same range of $x$.

the minimum of the three eigenvalues (the continuous line) jumps from one eigenvalue to another (dashed lines). The regions in which it is negative belongs to different eigenvalues depending on the twisted sector charge and agrees with the closed string prediction for the existence of instabilities.

It was derived earlier 13 that a non-supersymmetric orbifold gauge theory with a fixed point, i.e. with $x_{3}=1-x_{2}$, has no stable twisted sectors. We can easily identify this situation in Figure 1. For $0<x_{1}<1$ and $x_{3}=1-x_{2}$, we always have $1<x_{1}+x_{2}+x_{3}<2$.

\subsection{A no-go theorem}

Now we are ready to prove a "no-go theorem" for non-supersymmetric conformal orbifolds of $\mathcal{N}=4 \mathrm{SYM}$. Let us assume that we have a nonsupersymmetric orbifold and focus on an element $g$ which, after being diagonalized, becomes $r(g)=\operatorname{diag}\left(e^{2 \pi i x_{1}}, e^{2 \pi i x_{2}}, e^{2 \pi i x_{3}}, e^{-2 \pi i X}\right)$ with $X=x_{1}+x_{2}+x_{3} \bmod 1, x_{i} \neq 0$ for all $i$ and $x_{1}+x_{2}+x_{3} \neq 0 \bmod 1$. In the appendix we argue that, in agreement with the intuition, such an element always exists. As we have seem in section 4 , if $1<x_{1}+x_{2}+x_{3}<2$ this sector is unstable. The remaining two cases, $0<x_{1}+x_{2}+x_{3}<1$ and $2<x_{1}+x_{2}+x_{3}<3$, which are related by the transformation $g \rightarrow g^{-1}$ require us to consider the subgroup of the orbifold group $\Gamma$ generated by $g$. Since 


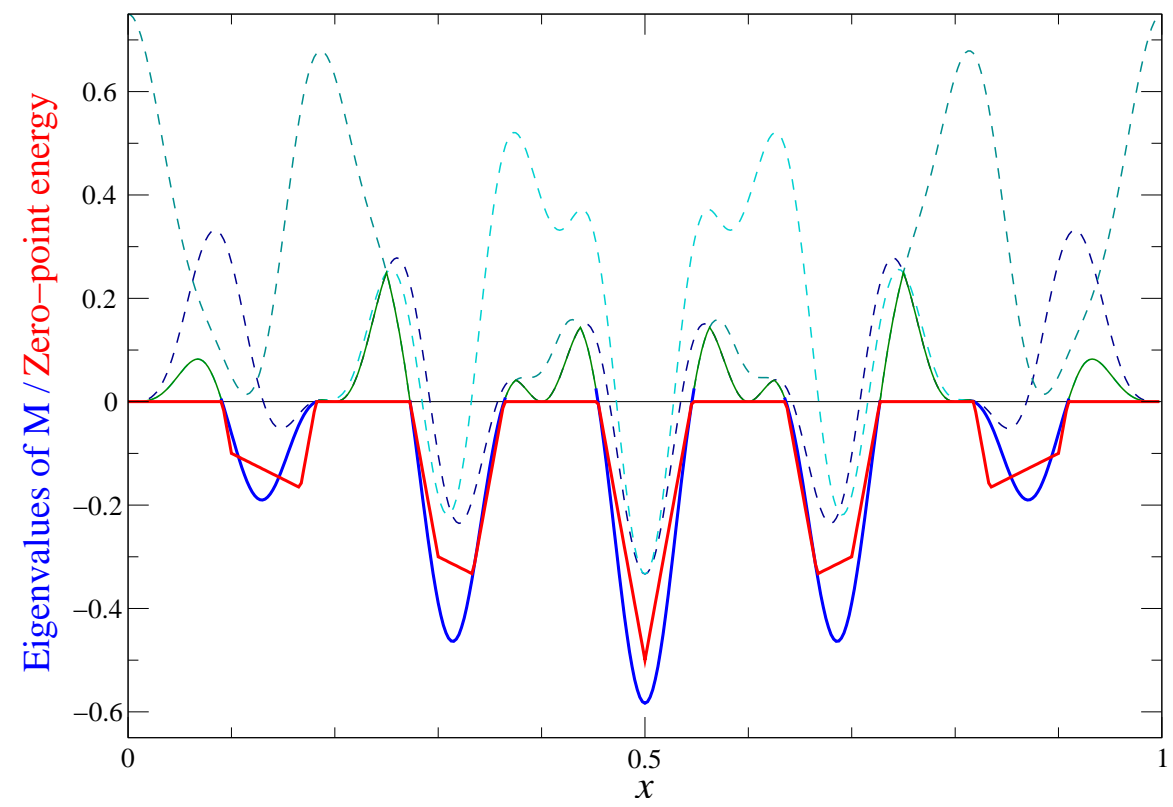

Figure 4: The (rescaled) eigenvalues of the discriminant matrix, the smallest eigenvalue (continuous line) and the closed string zero-point energy as a function of normalized twisted sector charge $x$ for an $S U(2)$-symmetric orbifold. The crossing of eigenvalues as a function of charge is cricial for the successful match of gauge theory and string theory instabilities.

the orbifold group is assumed to be finite, this subgroup is isomorphic to $\mathbb{Z}_{k}$ with some finite $k$. If the generator of this $\mathbb{Z}_{k}$ has all nonvanishing $x_{i}=m_{i} / k$ and $0<x_{1}+x_{2}+x_{3}<1$, the element $g^{n}$ with a sufficiently large $n$ is such that $1<n\left(x_{1}+x_{2}+x_{3}\right)<2$ and thus potentially yields an unstable sector.

A subtlety which requires a more detailed discussion stems from the fact that sometimes $n x_{i}$ or $n X$ are integers and the corresponding group element preserves supersymmetry. Let us recall the partition of the domain of $\left(x_{1}, x_{2}, x_{3}\right)$ introduced in section 4 . We called the part of the cube $x_{1}+x_{2}+x_{3} \leq 1$ (with all edges included i.e. with planes $x_{1}=0, x_{2}=0$, $x_{3}=0, X=1$ ) the "first" region, the part $2 \leq x_{1}+x_{2}+x_{3}<3$ (with only one edge $x_{1}+x_{2}+x_{3}=2$ included) the "second" region and the area $1<x_{1}+x_{2}+x_{3}<2$ (without any edges included) the "negative" region.

The element $g$ described above is just a point in the "first" region. Now let us consider an arbitrary point $g^{\prime}$ in the "first" region. We assume it is also from our $\mathbb{Z}_{k}$. The multiplicative group operation translates into an additive operation at the level of the parameters $x_{i}^{g}$ : $x_{i}^{g^{\prime}+g}=x_{i}^{g^{\prime}}+x_{i}^{g}$. The crucial observation is that, due to the fact that $x_{i}<1$, the point $g^{\prime}+g$ cannot lie in the "second" region for any $g$ and $g^{\prime}$ from the "first" region. The point $g+g^{\prime}$ could be in the "first" or "negative" region only. 
This observation is actually enough to prove the "no-go theorem." We start with a point in the "first" region, corresponding to $g$, but must finish in the "second" region, corresponding to $g^{k-1}=g^{-1}$. Since, as we argued above, we can not jump directly from the "first" to the "second" without being in the "negative" region at least once, there is at least one group element which corresponds to a point in the "negative" region and the corresponding sector is unstable. It therefore follows that no non-supersymmetric orbifold of $\mathcal{N}=4 \mathrm{SYM}$ is conformal in the one-loop treatment.

\section{Discussion}

In motivating the AdS/CFT correspondence, it is customary to begin with the 3-brane metric

$$
h^{-1 / 2} d x^{2}+h^{1 / 2}\left(d r^{2}+r^{2} d \Omega^{2}\right),
$$

containing the warp factor $h(r)=1+L^{4} / r^{4}$, and then taking a decoupling limit where $r^{4} \ll L^{4}$ so that the constant term in the warp factor is neglected. Since $L^{4}=a \lambda$, where $a$ is a proportionality constant, the dual of a weakly coupled gauge theory is a highly curved space $A d S_{5} \times S^{5} / \Gamma$ of small radius $L$. The spectrum of closed strings in such a background is difficult to compute reliably.

But let us imagine taking $\lambda$ to be small before the decoupling limit is taken. Then, the back-reaction of the stack of $N$ D3-branes is small, and we can expand $h^{1 / 2}(r)=1+$ $\frac{a \lambda}{2 r^{4}}+\ldots$ in the metric. The leading term in this expansion corresponds to a closed string background $\mathbb{R}^{3,1} \times \mathbb{R}^{6} / \Gamma$ where the spectrum of closed strings can be studied with ease. Of course, a suggestion that closed string theory on this background is somehow dual to the weakly coupled orbifold gauge theory seems impossible in general. But perhaps the twisted sector states, localized near the tip of the cone, do incorporate some features of the weakly coupled gauge theory (this is to some extent suggested by the research on localized tachyons 18, 20, 21, 22]). What we have found in this paper is that the stability/instability of this background is actually in one-to-one correspondence with the stability/instability of the orbifold gauge theory on $N$ D3-branes placed at the tip of the cone. What are the reasons for this agreement? One possibility is that the stability is a "topological" observable that is not affected by taking various limits, i.e. the stability can be studied reliably even before the decoupling limit is taken. Our results indeed suggest that the standard arguments for the decoupling of low-energy gauge theory on $N$ D3-branes at the tip of the cone from closed string modes fail when the closed string spectrum contains tachyons, either localized or bulk. Clearly, we need a better understanding of the effect of twisted closed string modes on the low-energy gauge theory.

It would also be interesting to use our new results to study the closed string tachyon 
condensation in more detail. At least for the non-freely acting orbifolds, its gauge theory dual [12, 10] is the Coleman-Weinberg mechanism [23] where single-trace operators charged under $\Gamma$ acquire expectation values, thus breaking the quantum symmetry of the orbifold gauge theory. It would be interesting to study whether a similar mechanism is at work also for the orbifolds that are freely acting on the 5-sphere; for such orbifolds the tachyons are localized at the tip of the cone. Finding relations with existing research on localized tachyon condensation (see, for example, [18, 20, 21, 22]) may lead to progress in this direction, as well as to possible insight into the phase transition and subsequent tachyon condensation in the small radius regime of $A d S_{5} \times S^{5} / \Gamma$. The analysis of localized tachyon condensation on $\mathbb{C}^{3} / \mathbb{Z}_{n}$ led to the conclusion that in type II theories the singularity is completely resolved 24]. Turning on the tachyon vev was identified in a probe-brane theory with turning on twisted Fayet-Iliopoulos terms. Given the surprising agreement we found, it is possible that turning on a tachyon vev below the phase transition radius has a similar interpretation. In fact, the double-trace deformations we needed to introduce at tree level for general abelian orbifold theories off the natural line arise from integrating out the auxiliary fields descending from the $\mathcal{N}=4 D$ auxiliary fields. As in the probe-brane analysis, turning on twisted Fayet-Iliopoulos terms corresponds to further deforming the action by the operators $O^{g}$. In the gauge theory, this is actually a simple consequence of a twisted operator developing a vev: when a double-trace operator $O^{g} O^{g^{\dagger}}$ is present in the action and $O^{g^{\dagger}}$ condenses, this effectively deforms the action by a single-trace operator $O^{g}$. In the presence of such a deformation the features of the $\mathrm{RG}$ flow are quite different compared to the $\Gamma$-symmetric case. In particular, below the scale of the condensate, the gauge coupling constant acquires a nontrivial beta function. Matching the IR properties of such a deformed gauge theory with the localized tachyon condensation is an interesting problem.

Another interesting direction to pursue is the identification of "subleading instabilities" in the gauge theory, which are due to higher eigenvalues of $M$ crossing zero, with excited closed strings becoming tachyonic. As we showed for the class of $S U(3)$ symmetric orbifolds, the onset of the octet instability as a function of $x$ is numerically very close to the value of $x$ where an excited free closed string becomes tachyonic. That it is not exactly the same could be due to the fact that a singlet tachyon is already condensed, which has not been taken into account in our calculations. Perhaps this issue could be studied in more detail.

\section{Acknowledgments}

We are grateful to A. Parnachev and E. Weinberg for useful discussions. A. D. and R. R. would like to thank the Third Simons Workshop in Mathematics and Physics, where a part of this work was done. Some of I. R. K.'s work on this project took place at the Aspen Center for Physics, which he thanks for hospitality. The research of A. D. is supported in 
part by grant RFBR 04-02-16538 and by the National Science Foundation Grant No. PHY0243680. The research of I. R. K. is supported in part by the National Science Foundation Grant No. PHY-0243680. The research of R. R. was supported in part by funds provided by the U.S.D.O.E. under co-operative research agreement DE-FC02-91ER40671. Any opinions, findings, and conclusions or recommendations expressed in this material are those of the authors and do not necessarily reflect the views of the National Science Foundation. 


\section{A. A nonsupersymmetric element of nonsupersymmetric orbifold}

To complete the proof in section 6.1 we now argue that, if the orbifold group breaks supersymmetry, there always exists an element $g$ of $\Gamma$ which acts nontrivially on all spinors. This is obviously true if the orbifold group is abelian: since the orbifold breaks all supersymmetries it follows that, after diagonalizing all group elements, different elements of $\Gamma$ preserve different spinors. Then, the desired group element $g$ is simply the product of sufficiently many elements of $\Gamma$ preserving spinors. If each group generator preserves one spinor it is typically sufficient to simply multiply two such generators. Special care must be taken when some spinor has opposite charges with respect to the two generators:

$$
g_{1}|\psi\rangle=e^{i x}|\psi\rangle \quad g_{2}|\psi\rangle=e^{-i x}|\psi\rangle \quad \rightarrow \quad g_{1} g_{2}|\psi\rangle=|\psi\rangle .
$$

If this is the case it suffices to raise one of the generators to some power different from unity. It is not hard to see that this leads to the desired result. Indeed, given our assumption we have to analyze only two charges for each generator; we denote them as the ordered pairs $\left(x_{2}^{(1)}, x_{3}^{(1)}\right)$ and $\left(x_{2}^{(2)}, x_{3}^{(2)}\right)$. If $x_{2}^{(1)}=-x_{2}^{(2)}$ it is necessary to find an integer $n$ such that $n\left(x_{2}^{(1)}, x_{3}^{(1)}\right)+\left(-x_{2}^{(1)}, x_{3}^{(2)}\right) \neq(0,0) \bmod 1$. Any integer different from $-\left[x_{3}^{(2)} / x_{3}^{(1)}\right]$ has the desired effect.

The situation is less transparent if the orbifold group is nonabelian, as the generators cannot be simultaneously diagonalized. The goal is to show that, starting from a generator $g_{1}$ preserving a spinor $|\psi\rangle$, it is possible to find a group element $g_{2}$ preserving a different spinor such that for some $n \in \mathbb{Z}, g_{2}^{n} g_{1}$ preserves no spinor. Without loss of generality we can pick $g_{1}$ to be diagonal. We can also assume that $g_{2}$ has one unit eigenvalue (if it did not then $g_{2}$ is the group element we are looking for) and that the spinor preserved by $g_{2}$ is different from the spinor preserved by $g_{1}$ (if no such $g_{2}$ exists then $\Gamma$ preserves supersymmetry). The condition that $g_{2}$ belongs to a subgroup of $S U(4)$ implies then that only two of its eigenvalues are independent. We can always write $g_{2}$ as $g_{2}=v g_{2}^{d} v^{\dagger}$, where $v$ is some unitary matrix and $g_{2}^{d}$ is diagonal.

The condition that the product $g_{2} g_{1}$ has one unit eigenvalue

$$
\operatorname{det}\left(g_{2} g_{1}-\mathbb{1}\right)=0
$$

can be thought of as giving an additional relation between the eigenvalues od $g_{2}$. We distinguish two cases.

1) If no orbifold group element (not necessarily a generator, and except $g_{1}^{k}$ for all $k$ ) satisfies this relation then we can pick any one of them as $g_{2}$.

2) If there exists one group element such that the equation above is satisfied, then

$$
\operatorname{det}\left(g_{2}^{n} g_{1}-\mathbb{1}\right)=0
$$


is not satisfied for some $n$. Indeed, since for any $n g_{2}^{n}$ is diagonalized by the same matrix $v$ as $g_{2}$, we can think of this equation as providing additional constraints on the eigenvalues of $g_{2}$. Since under our assumptions $g_{2}$ has only two independent eigenvalues, this equation can be satisfied only for one value of $n \neq 1$. If this value exists it is sufficient to pick a value of $n$ different from it.

Thus, if $g_{2}$ generates a group larger than $\mathbb{Z}_{3}$, it is always possible to find an element in $\Gamma$ which does not leave any spinor invariant. For subgroups of $S U(4)$ for which each element preserving some spinor generates a $\mathbb{Z}_{k}$ with $k \leq 3$ a separate analysis is needed. Luckily there are few such groups [19] and it is straightforward though quite tedious to analyze them one by one. Even in these cases it continues to be true that there always exists an element of $\Gamma$ with no invariant spinors. 


\section{References}

[1] J. M. Maldacena, "The large N limit of superconformal field theories and supergravity," Adv. Theor. Math. Phys. 2, 231 (1998) [Int. J. Theor. Phys. 38, 1113 (1999)] arXiv:hep-th/9711200.

[2] S. S. Gubser, I. R. Klebanov and A. M. Polyakov, "Gauge theory correlators from non-critical string theory," Phys. Lett. B 428, 105 (1998) arXiv:hep-th/9802109.

[3] E. Witten, "Anti-de Sitter space and holography," Adv. Theor. Math. Phys. 2, 253 (1998) arXiv:hep-th/9802150.

[4] O. Aharony, S. S. Gubser, J. M. Maldacena, H. Ooguri and Y. Oz, "Large N field theories, string theory and gravity," Phys. Rept. 323, 183 (2000) arXiv:hep-th/9905111.

[5] I. R. Klebanov, "TASI lectures: Introduction to the AdS/CFT correspondence," arXiv:hep-th/0009139

[6] S. Kachru and E. Silverstein, "4d conformal theories and strings on orbifolds," Phys. Rev. Lett. 80, 4855 (1998) arXiv:hep-th/9802183.

[7] A. E. Lawrence, N. Nekrasov and C. Vafa, "On conformal field theories in four dimensions," Nucl. Phys. B 533, 199 (1998) arXiv:hep-th/9803015.

[8] M. Bershadsky, Z. Kakushadze and C. Vafa, "String expansion as large N expansion of gauge theories," Nucl. Phys. B 523, 59 (1998) arXiv:hep-th/9803076;

[9] M. Bershadsky and A. Johansen, "Large N limit of orbifold field theories," Nucl. Phys. B 536, 141 (1998) arXiv:hep-th/9803249.

[10] A. A. Tseytlin and K. Zarembo, "Effective potential in non-supersymmetric $\mathrm{SU}(\mathrm{N}) \mathrm{x}$ $\mathrm{SU}(\mathrm{N})$ gauge theory and interactions of type 0 D3-branes," Phys. Lett. B 457, 77 (1999) arXiv:hep-th/9902095.

[11] C. Csaki, W. Skiba and J. Terning, "Beta functions of orbifold theories and the hierarchy problem," Phys. Rev. D 61, 025019 (2000) arXiv:hep-th/9906057.

[12] A. Adams and E. Silverstein, "Closed string tachyons, AdS/CFT, and large N QCD," Phys. Rev. D 64, 086001 (2001) arXiv:hep-th/0103220.

[13] A. Dymarsky, I. R. Klebanov and R. Roiban, "Perturbative search for fixed lines in large N gauge theories," arXiv:hep-th/0505099. 
[14] A. Armoni, E. Lopez and A. M. Uranga, "Closed strings tachyons and non-commutative instabilities," JHEP 0302, 020 (2003) arXiv:hep-th/0301099.

[15] S. Frolov, "Lax pair for strings in Lunin-Maldacena background," arXiv:hep-th/0503201

[16] E. Fuchs, "The U(1)s in the finite N limit of orbifold field theories," JHEP 0010, 028 (2000) arXiv:hep-th/0003235.

[17] I. R. Klebanov, N. A. Nekrasov and S. L. Shatashvili, "An orbifold of type 0B strings and non-supersymmetric gauge theories," Nucl. Phys. B 591, 26 (2000) arXiv:hep-th/9909109.

[18] A. Adams, J. Polchinski and E. Silverstein, "Don't panic! Closed string tachyons in ALE space-times," JHEP 0110, 029 (2001) arXiv:hep-th/0108075.

[19] A. Hanany and Y. H. He, "A monograph on the classification of the discrete subgroups of SU(4)," JHEP 0102, 027 (2001) arXiv:hep-th/9905212.

[20] J. A. Harvey, D. Kutasov, E. J. Martinec and G. W. Moore, "Localized tachyons and RG flows," arXiv:hep-th/0111154.

[21] E. J. Martinec, "Defects, decay, and dissipated states," arXiv:hep-th/0210231.

[22] M. Headrick, S. Minwalla and T. Takayanagi, "Closed string tachyon condensation: An overview," Class. Quant. Grav. 21, S1539 (2004) arXiv:hep-th/0405064.

[23] S. R. Coleman and E. Weinberg, "Radiative Corrections As The Origin Of Spontaneous Symmetry Breaking," Phys. Rev. D 7, 1888 (1973).

[24] D. R. Morrison, K. Narayan and M. R. Plesser, JHEP 0408, 047 (2004) arXiv:hep-th/0406039. 